\title{
New Spread Spectrum Techniques for Multiple Antenna Transmit Diversity
}

\author{
Dinesh Rajan, Elza Erkip and Behnaam Aazhang \\ Department of Electrical and Computer Engineering, \\ Rice University, 6100 S.Main Street, \\ Houston, TX 77005. \\ dinesh@rice.edu, elza@rice.edu, aaz@rice.edu
}

\begin{abstract}
We propose a new scheme for multiple antenna transmission in the context of spread spectrum signaling. The new scheme consists of using shifted Gold sequences to modulate independent information on the multiple antennas. We show that this new scheme greatly improves the throughput over currently known multiple-antenna methods. We also find the optimal power allocation strategy among multiple transmit antennas for a fixed feedback rate.
\end{abstract}

\section{Preliminaries}

Multiple antennas are vital for downlink communications in future cellular systems to achieve higher bit rates and better quality of services. Wideband code division multiple access (CDMA) has been universally accepted as the preferred modulation scheme for the next generation cellular systems.

In this paper, we propose a new spreading method for multiple antenna transmission. We show that using different phases of the same spreading code on different antennas provides us with better performance and modulating independent information provides higher throughput.

It is well known that Gold codes have good crosscorrelation properties [3]. They are designed so that circularly shifted codes have low cross-correlation with the unshifted ones. We use this property to increase the throughput of the system without decreasing the number of users the system can support.

\section{New Transmission Scheme}

We consider the downlink case where the base station sends independent information to different mobiles. We assume the receiver can track the fading corresponding to signals coming form different antennas. We confine ourselves to direct sequence CDMA and use Gold codes for spreading purposes. In our scheme, we transmit two independent bit streams for each user through the two antennas, using different phases of the same spreading code. Hence we maintain the number of spreading codes available for different users the same as in the single antenna case.

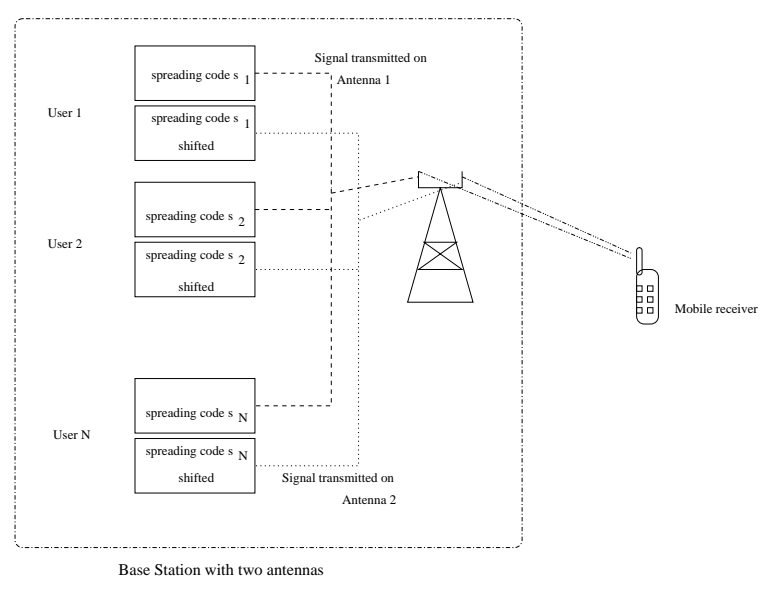

Figure 1: New Transmission Scheme

We assume the total transmit power allocated to a particular mobile is $\mathrm{P}$. When no feedback information about the fading is available at the transmitter, by symmetry, the bits on both the antennas receive half the power, that is each gets $P / 2$. Also as the spreading length is kept unchanged, we maintain the 
same bandwidth as in single antenna case.

The model of our new scheme is given in Figure 1. The transmitted signal on antenna $j$ at time $i$ is given by

$$
X_{j i}=\sum_{k=1}^{N} P_{k j} s_{k j} b_{k j i},
$$

where $P_{k j}, s_{k j}, b_{k j i}$ denote respectively the power, the spreading code and the bit belonging to the $k^{t h}$ user modulated on the $j^{t h}$ antenna at time i. Note that the new scheme with, say, two antennas will have $s_{k 1}$ as a Gold code, $s_{k 2}$ as the same Gold code shifted by a predetermined amount. Also, we will have

$$
P_{k 1}=P_{k 2}=P / 2
$$

The received signal at a particular mobile, is then given by

$$
Y_{i}=\sum_{j=1}^{2} X_{j i} A_{j i}+n_{i}
$$

where $A_{j i}$ is the fading amplitude at time $\mathrm{i}$ between antenna $\mathrm{j}$ and the mobile, $n_{i}$ is the additive white Gaussian noise with variance $\sigma^{2}$.

Since there are multiple users in the system, the mobile receiver uses a bank of chip matched filters followed by a decorrelator to extract its own information. We assume that the same diversity technique is used for all the mobiles. We analyze the system using a simple one shot decorrelator detector [7]. However we feel that the performance of our scheme will be similar with other detectors such as MMSE.

\section{Performance Analysis}

Many different transmit diversity schemes have been proposed for the next generation cellular systems such as orthogonal transmit diversity (OTD) [5], time switched transmit diversity (TSTD) [6] and selection transmit diversity (STD) [4]. Without loss of generality, we consider two transmit antennas and one receive antenna for all our analysis. In OTD, each user uses two different orthogonal spreading codes for transmission on the two antennas. To maintain the number of spreading codes available for each user the same as in single antenna case, the length of the spreading code is doubled. In TSTD, the same spreading code is used on both the antennas but at each time instant only one antenna is used.
The transmitter switches between the antennas in a preassigned manner.

In STD (also called maximal selection) the transmitter uses feedback information from the mobile. This feedback consists of 1 bit of information and indicates which of the two antennas has lower attenuation at every symbol instant. The transmitter then chooses the antenna with lowest attenuation to transmit the information.

A simple capacity analysis shows that OTD and TSTD have the same average throughput as a simple single antenna scheme. The main reason is the identical distribution of the fading process over the two antennas. Hence for the remainder of the paper, we will only provide comparisona with the single antenna scheme and maximal selection.

The probability of bit error [7] in the single antenna and maximal selection case are given, respectively, by

$$
\begin{aligned}
& P_{e-\text { single }}=Q\left(\frac{A \sqrt{P}}{\sigma \sqrt{\left(R^{-1}\right)_{11}}}\right) . \\
& P_{e-s t d}=Q\left(\frac{\max \left(A_{1}, A_{2}\right) \sqrt{P}}{\sigma \sqrt{\left(R^{-1}\right)_{11}}}\right) .
\end{aligned}
$$

where $R_{i j}=s_{i}^{t} s_{j} ; i, j=1, \ldots, N, s_{i}$ is the spreading code of the $i^{t h}$ user, $\mathrm{P}$ is the total power allocated to the particular mobile. Note that $\mathrm{R}$ is $N \times N$ where $\mathrm{N}$ is the number of users. For the new scheme the probability of bit error for the two independent bit streams transmitted on the two different antennas are given by

$$
\begin{aligned}
& P_{e 1}=Q\left(\frac{A_{1} \sqrt{P}}{\sqrt{2} \sigma \sqrt{\left(R^{-1}\right)_{11}}}\right) \\
& P_{e 2}=Q\left(\frac{A_{2} \sqrt{P}}{\sqrt{2} \sigma \sqrt{\left(R^{-1}\right)_{22}}}\right)
\end{aligned}
$$

Note that in the new scheme $\mathrm{R}$ incorporates the shifted as well as the unshifted spreading codes and has size $3 N \times 3 N$.

We compute the throughput of each system by modeling the channel between each antenna and the mobile as a Binary Symmetric Channel (BSC) with crossover probabilities given by the appropriate probabilities of error. The throughput of the single antenna case and in the maximal choosing case are given by

$$
\begin{gathered}
C_{\text {thro-single }}=E\left[1-H\left(P_{e}\right)\right] \\
C_{t h r o-s t d}=E\left[1-H\left(P_{e-s t d}\right)\right]
\end{gathered}
$$

where $H(p)$ is the binary entropy function [1] and the expectation is over the fading process. In the 
new scheme, since independent bits are transmitted through the two antennas, the throughput of the system is given by

$$
C_{\text {thro-new }}=E\left[2-H\left(P_{e 1}\right)-H\left(P_{e 2}\right)\right]
$$

We plot the throughput for the single antenna, maximal choosing and the new scheme in Figure 2 for 5 users using Gold codes of length 31 under Rayleigh fading. We assume that the fading processes between the two transmit antennas and the receiver, that is $A_{1}$ and $A_{2}$ are independent and identically distributed. The figure shows that our scheme per-

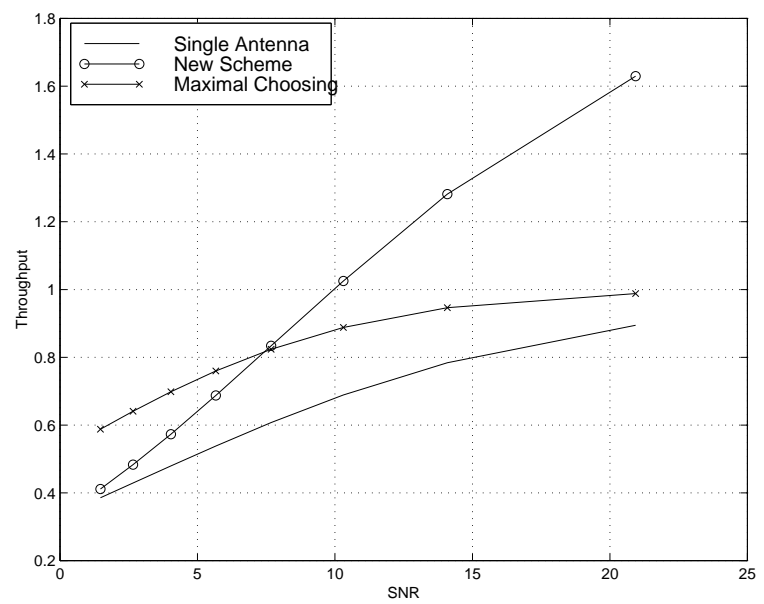

Figure 2: Throughput in bits/channel usage: 5 users, no correlation between antennas

forms very well at high SNR. Although the performance deteriorates for low SNR's we feel that in the range of SNR's required for normal operating bit error rates, the performance is satisfactory.

As the correlation between the antennas increases, the performance of maximal selection approaches the single antenna case, whereas our scheme has higher throughput.

The fact that our scheme is better than even maximal selection could be surprising at first. However this is in keeping with the arguments provided in [2] where it is proved that higher capacity is possible by having $\mathrm{N}$ users at spatially different locations than having one user of $\mathrm{N}$ times the power. Although the above argument was made for the uplink case, we could consider the two antennas at the base station to represent different users with powers $P / 2$. This would be better than having one user at power $\mathrm{P}$ on one of the antennas alone.

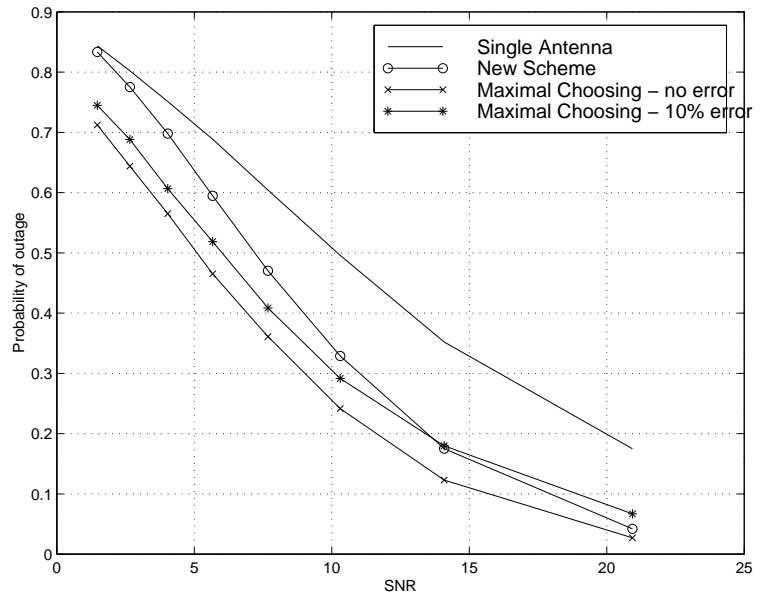

Figure 3: Probabilities of outage for rate $=0.9$

For the new scheme, the probability of outage, is given by the probability that the instantaneous rate falls below some specified rate R. The probability of outage can be expressed as:

$$
P_{\text {out }}=\operatorname{Pr}\left(\left[2-H\left(P_{e 1}\right)-H\left(P_{e 2}\right)\right]<R\right)
$$

We would like to compare this to the probability of outage for the single antenna and maximal choosing under independent Rayleigh fading on the two antennas. Note that for $\mathrm{R} \geq 1$, single antenna and maximal choosing have $P_{\text {out }}=1$, whereas the new scheme can still support rates higher than 1 bit/transmission. For $\mathrm{R}<1$, the maximal choosing has the advantage of knowing the best antenna and thus being less susceptible to variations. However, this comes at the expense of 1 bit of feedback from the mobile receiver.

It is interesting to observe the sensitivity of maximal choosing to errors in the feedback. In Figure 3, we plot $P_{\text {out }}$ vs $\mathrm{SNR}$ for $\mathrm{R}=0.9$ for single antenna, maximal choosing, maximal choosing with $10 \%$ error and the new scheme. We observe that maximal choosing is quite susceptible to feedback errors and for high SNR the new scheme has better performance than maximal choosing with feedback errors.

\section{Coded Schemes}

In the previous section we showed that the new scheme is capable of supporting higher rate information transmission. Alternatively, we can keep the data rate constant and use the new scheme to improve the probability of bit error. We consider a 
simple 'outer' coding scheme in which the data that was transmitted in the single antenna case which is already coded is passed through a rate $1 / 2$ convolutional encoder. We multiplex the resulting coded bit stream and send it on the two different antennas using the new transmission scheme. The plot

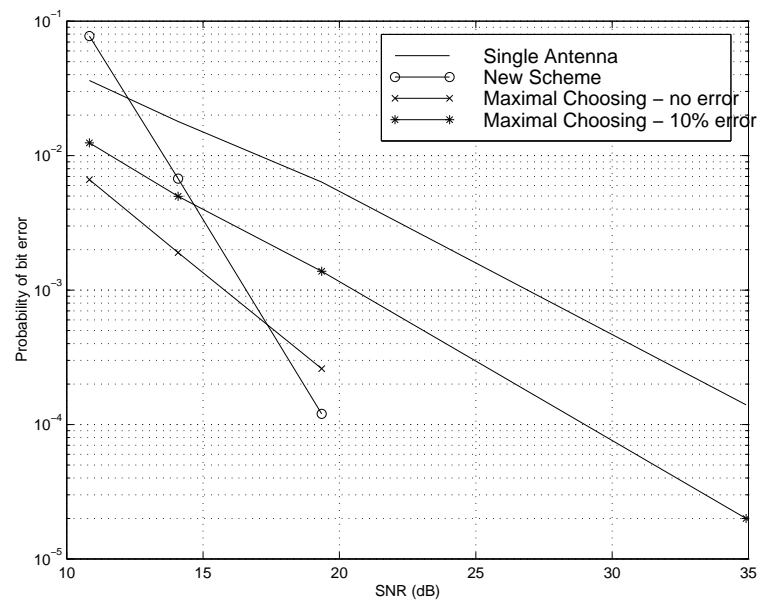

Figure 4: Bit Error Rates of single antenna, new scheme and maximal choosing

of bit error rates for our scheme along with single antenna and maximal selection are given in Figure 4. The results indicate that our scheme performs very well as compared to the single antenna case. This shows that the increased dimensionality due to the presence of multiple antennas can also be used to provide stronger channel coding. Also when compared to maximal choosing our scheme performs well for high SNR. As the feedback error increases in maximal choosing, the performance becomes worse than the new scheme for lower SNR's as well. In the case of the original bit stream being channel coded one can get even better performance from the new scheme by combining the channel coding that is already present with the 'outer' coding.

\section{Optimal Power Allocation}

An analysis of Figure 2 shows that for low SNR values maximal choosing performs better than the new strategy whereas for high SNR the performance comparison is reversed. One way to understand the difference between these two schemes is to consider the power allocated to the two antennas in the two cases. In maximal choosing the power allocated to the two antennas is either $[\mathrm{P}, 0]$ or $[0, \mathrm{P}]$, whereas in the new scheme it is $[P / 2, P / 2]$. We proceed to find the optimum power allocation to the two antennas $[\alpha P,(1-\alpha) P], 0 \leq \alpha \leq 1$ based on 1 bit of feedback information from the mobile receiver. We assume that this 1 bit of feedback will inform the transmitter about which antenna has lower attenuation. Note that maximal choosing corresponds to $\alpha=1$ or 0 whereas the new strategy (which was designed for a no-feedback case) has $\alpha=1 / 2$. For this optimization we assume the modulation is restricted to BPSK.

The total throughput in this case is given by

$$
\begin{gathered}
C_{\text {thro }}= \\
E\left[2-H\left(Q\left(A_{1} \sqrt{\alpha S N R}\right)\right)-H\left(Q\left(A_{2} \sqrt{(1-\alpha) S N R}\right)\right)\right]
\end{gathered}
$$

where $\alpha$ is the power allocated to the 'good' antenna. The optimum value of $\alpha$ is dependent on the operating SNR of the system and is shown in Fig. 5 for the case of antennas correlated by 0.75 . The plot of the performance characterized by the throughput is given in Fig. 6 We observe that for low SNR the opti-

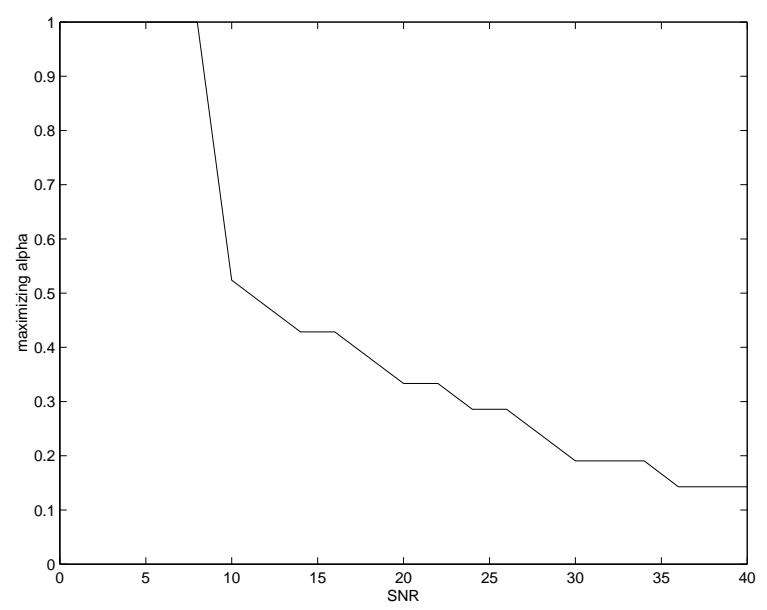

Figure 5: Optimal Power distribution between antennas for a non-orthogonal system for various SNR

mal power allocation scheme gives considerable gain over equal power allocation to the two antennas. In fact the optimizing $\alpha$ is 1 which corresponds to maximal choosing. However, we would like to stress once again that this comes at the expense of 1 bit accurate feedback from the receiver to the transmitter. As the SNR increases, this value of the maximizing $\alpha$ decreases. Asymptotically as the SNR reaches infinity, the maximizing $\alpha$ reaches zero. This can be easily explained by the fact that the marginal gain is 
more in the 'worse' antenna for high SNR because of the concavity of the performance curve as a function of SNR. We also observe that even though maximizing $\alpha$ is different than $1 / 2$ for large SNR, the performance obtained by $\alpha=1 / 2$ (as suggested by our new strategy) is very close to optimal. Of course, choosing $\alpha=1 / 2$ requires no feedback, and hence is a lower complexity strategy.

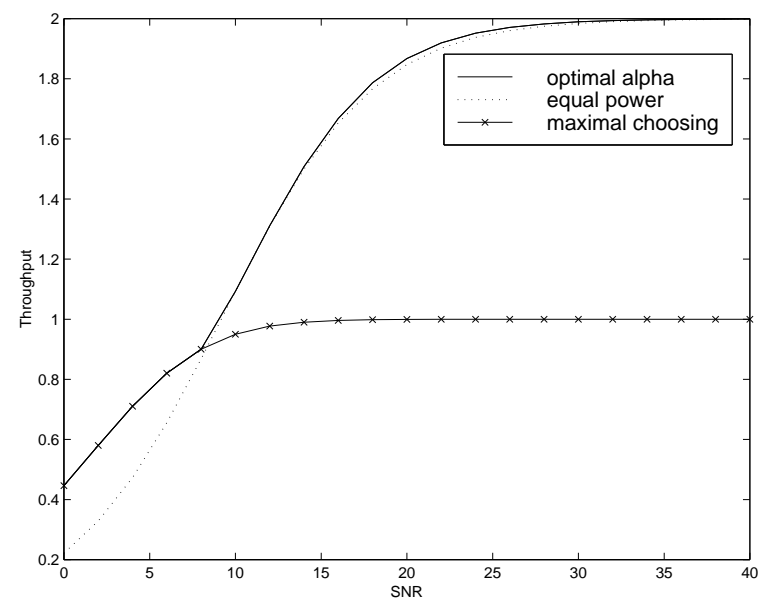

Figure 6: Throughput under optimal power distribution, equal power sharing and maximal choosing, non-orthogonal codes

\section{Summary}

We have proposed a new scheme for transmitting independent information on multiple antennas using different phases of Gold codes on different antennas. This scheme can also be applied to the case of higher number of antennas to get even more improvement in throughput and outage. Note, however, that this might increase the hardware complexity. The new scheme can be easily disabled if the mobile does not support extra processing by reallocating the power between the two phases of spreading codes.

In practice, although the two antennas at the base station are physically separated, the fading processes might still have some correlation. We have shown that the new scheme performs well for correlated fading as well. We expect the performance of this scheme using other kinds of detectors like MMSE to show similar trends. We have also found the optimal power distribution strategy amongst multiple antennas.

\section{References}

[1] T. M. Cover and J. A. Thomas. Elements of Information Theory. Wiley, New York, 1991.

[2] Robert G. Gallager. An inequality on the capacity region of multiaccess multipath channels. In Communications and Cryptography - Two sides of one Tapestry, pages 129-139. Kluwer, 1994.

[3] John G. Proakis. Digital Communications. McGraw Hill Inc., 1995.

[4] James A. Ritchey and Murat Azizoglu. Impact of switching constraints on selection diversity performance. 32 ${ }^{\text {nd }}$ Asilomar Conference on Signals, Systems and Computers, pages 795-800, November 1998.

[5] Kamyar Rohami. Orthogonal transmit diversity (OTD) for CDMA forward link. Motorola Contribution to ETSI, 1997.

[6] Samsung. Time switched transmit diversity (TSTD). Contribution by TTA to TIA-TTA JSG/01.98.05.13.05, 1998.

[7] Sergio Verdu. Multiuser Detection. Cambridge University Press, 1998. 